\title{
Effect of acupuncture and its influence on cerebral activity in patients with persistent asthma: study protocol for a randomized controlled clinical trial
}

Siyi Yu

Chengdu University of Traditional Chinese Medicine

Xiaohui Dong

Chengdu University of Traditional Chinese Medicine

Ruirui Sun

Chengdu University of Traditional Chinese Medicine

Zhaoxuan He

Hospital of Chengdu University of Traditional Chinese Medicine

Chuantao Zhang

Chengdu University of Traditional Chinese Medicine

Mei Chen

The Fifth People's Hospital of Chengdu

\section{Xiaojuan Hong}

Chengdu University of Traditional Chinese Medicine

Lei Lan

Chengdu University of Traditional Chinese Medicine

Fang Zeng ( $\square$ zengfang@cdutcm.edu.cn )

Chengdu University of Traditional Chinese Medicine

\section{Study protocol}

Keywords: Acupuncture, Asthma, meridians-viscera relationship, Functional magnetic resonance imaging, Central mechanism

Posted Date: April 1st, 2020

DOI: https://doi.org/10.21203/rs.2.21010/v2

License: (1) (1) This work is licensed under a Creative Commons Attribution 4.0 International License.

Read Full License 
Version of Record: A version of this preprint was published at Trials on May 14th, 2020. See the published version at https://doi.org/10.1186/s13063-020-04319-w. 


\section{Abstract}

Background Previous studies suggested that acupuncture was a promising adjunct treatment for asthma. However, the underlying mechanism of acupuncture for asthma remains unclear. The present trial aimed to explore whether and how specific meridian acupuncture works in quality of life and symptomatic improvement by modulating brain function in asthma patients. Methods : A randomized controlled functional brain imaging trial is currently being conducted in Sichuan, China. In total 48 patients with mild to moderate, persistent asthma will be recruited randomly allocated to two acupuncture groups: acupuncturing at Lung Meridian and acupuncturing at Heart Meridian . The treatment period will last for 4 weeks. The Asthma quality of life questionnaire (AQLQ) is the primary outcome. Asthma control test (ACT), the rate of Peak expiratory flow (PEFR), Forced expiratory volume in 1 second (FEV1), Montreal cognitive assessment (MoCA), Self-rating Anxiety Scale (SAS) and Self-rating Depression Scale (SDS) will also assess the clinical efficacy of different interventions. Functional magnetic resonance imaging (fMRI) scans will be performed to detect cerebral activity changes in each group. The clinical data and fMRI data will be analyzed, respectively. Pearson correlation analysis will be used to assess the association between the changes of cerebral activity features and the improvement of clinical outcomes in each group. Discussion : The present study has been established on the "meridians-viscera relationship" theory of traditional Chinese medicine and modern central mechanism of acupuncture. The results of this trial would be useful to identify the efficiency of the specific meridian acupuncture for asthma. The investigation of its central mechanism would further expand our knowledge of the acupuncture for asthma. Trial registration: Chinese Clinical Trial Registry, ChiCTR1900027478. Registered on 15 November 2019.

\section{Background}

Asthma is a chronic respiratory disease that affects about 300 million people worldwide or $4.3 \%$ of the world's population. More than $40 \%$ of patients with asthma are thought to have persistent symptoms that need long-term controller therapy [1-3]. The goals of asthma control are to minimize both the risk of adverse asthma outcomes and the symptom burden[3]. Most patients with mild to moderate asthma can be well controlled by medications such as inhaled corticosteroids (ICS), either alone or in combination with a long-acting $\beta 2$ agonist (LABA) [3]. However, patients with mild to moderate asthma remain at risk of asthma exacerbations, and manifest considerable heterogeneity in asthma symptom control and health-related quality of life [4]. Thus, seeking complementary and alternative interventions abstract both practioners and patients [3].

Accumulating evidence indicates that acupuncture treatment can both significantly improve asthmarelated quality of life (QoL) and lung function, and decrease medication dosages for asthmatic patients [5-10]. A recent meta-analysis, including nine randomized controlled trials (RCTs) with a total of 777 patients, demonstrated that the symptom response rate of conventional treatments plus acupuncture for asthma is statistically higher than conventional treatments alone [11]. These studies suggest that 
acupuncture may be a promising complementary option for asthma. However, the underlying mechanism of acupuncture for asthma remains unclear, which has limited its application in asthma control.

Although asthma results from airway inflammation, using magnetic resonance imaging (MRI) technique, people found that asthma is also characterized by cerebral structural and functional changes in some specific areas/circuits [12-17]. For example, using diffusion tensor imaging (DTI), Gao et al. had found that the patients with asthma shown an abnormal structure in multiple white matter networks including the fronto-limbic network, and the significant correlation was found between these abnormal structural findings and clinical symptom [17]. By functional MRI, the investigator found that the asthmatic patients manifested increased activity in the anterior cingulate cortex (ACC) and insula, and the functional alternations in these brain regions are associated with the degree of lung inflammation [14]. These findings remarkably enhanced our understanding of the central pathophysiological mechanisms of asthma, and provide the possibility and necessity to explore the central mechanism of acupuncture for asthma control.

With the guidance of the theory on the meridian-viscera association of traditional Chinese acupuncture, asthma has been recognized as a pulmonary disease and Lung Meridian is the most frequently used meridian for successful treatment of asthma. Therefore, The aims of this study are as follows: (i) assess the therapeutic effects of acupuncture for persist asthma by comparing acupuncturing at specific meridian (Lung Meridian) and no-specific meridian (e.g. Heart Meridian); (ii) explore the potential central mechanism of acupuncture for asthma using fMRI technique; (iii) investigate the possible correlation between brain activity changes elicited by different acupuncture interventions and the symptom and QoL improvements.

\section{Methods/design}

This is a randomized controlled neuroimaging trial which will be performed at the First Affiliated Hospital of Chengdu University of Traditional Chinese Medicine (CDUTCM). A total of 48 patients with persistent asthma will be recruited and randomly allocated in a 1:1 ration to two acupuncture groups: group $A$ (puncturing at acupoints on the Lung Meridian) and group B (puncturing at acupoints on the Heart Meridian). The treatment period will last for 4 weeks. Clinical outcome measurements and fMRI scans will be assessed at baseline and the end of treatment. The details of the study design are shown in Figure 1.

The study protocol conforms to the SPIRIT 2013 statement (Standard Protocol Items: Recommendations for Interventional Trials) [18]. The study has been approved by the Institutional Review Board (IRB) the First Affiliated Hospital of CDUTCM (approved number. 2019KL-045) and registered at Clinical Trial Registry (registration number: ChiCTR1900027478). All participants will provide voluntary written informed consent after a full discussion about the potential benefits and risks before participation. On the consent form, participants will be asked if they agree to use of their data should they choose to withdraw from the trial. Participants will also be asked for permission for the research team to share relevant data 
with people from the Universities taking part in the research or from regulatory authorities, where relevant. This trial does not involve collecting biological specimens for storage. YSY and XHD will recruiting the patient and gaining informed consent before participation.

\section{Participants}

\section{Recruitment strategies}

Asthma patients will be recruited mainly from the outpatient clinic in the respiratory department of the First Affiliated Hospital of CDUTCM. Potential patients will also be recruited via advertisement, leaflets and social media. All potential asthma patients will take a physical examination and laboratory tests, such as lung function test, $x$-ray plate, routine blood test, and blood immune test (immunoglobulin $E$, IgE). A respiratory physician will make the final diagnosis of potential patients. The diagnosis of asthma according to Western Medicine definition, not use with a specific TCM pattern.

\section{Inclusion criteria}

Eligible participants should fulfill all of the following items: (1) belonging to mild to moderate, persist asthma, according to the Chinese guideline for the prevention and management of bronchial asthma (version 2016) [19]; (2) aging from 18 to 65 years; (3) right-handed; (4) not participating in any other clinical trials in the past one month; (5) having assigned informed consent.

\section{Exclusion criteria}

Patients matching any of the following items will be excluded: (1) being diagnosed as other lung diseases, such as bronchiectasis, tuberculosis, lung abscess, cystic fibrosis, a- 1 antitrypsin deficiency, and restrictive lung disease, etc.; (2) having aggravating malignant tumors or other clinically significant diseases that would jeopardize patient safety, such as uncontrolled heart-failure, severe hypertension, uncontrolled arrhythmias, etc.; (3) occurring only when accidentally exposed to allergen or chemical sensitizer; (4) being pregnant, lactating or those with childbearing requirements for nearly half a year; (5) receiving acupuncture for asthma within the last four weeks; (6) having MRI contraindications, such as having a heart pacemaker, having metallic foreign bodies, having severe claustrophobia, etc.

\section{Sample size}

The sample size calculation of neuroimaging study is different from that of classic randomized controlled clinical trials. Power analyses for neuroimaging studies rely upon assumptions about blood oxygenation level-dependent (BOLD) signal amplitude, smoothness, brain location, and other factors that render principled a priori designations difficult. It has been suggested that for fMRI studies, a minimum sample size $(n=20)$ participants should be used in order to obtain $80 \%$ power with an error threshold of 0.002 at a single voxel level [20]. Considering a $20 \%$ dropout rate, the sample size in this study was increased to 24 per group, making a total of 48 . 


\section{Randomization}

After the baseline assessment, the eligible participants will be randomly allocated with an equal rate to their respective groups. To avoid bias on researchers 'subjective factors, randomization will be implemented by a clinical information management system (Beijing Bioknow Information Science \& Technology Co. Ltd., China). When participant-recruiting staffs decide to recruit an eligible asthma patient, they will send the patient's name, gender, age, and telephone numbers to this system online. And then, the randomized result will be delivered to the acupuncturists.

\section{Blinding}

Because of the different acupoints in two groups, the acupuncturists will not be blinded from the group allocation. Patients in two groups will be separated into cubicles to refrain from communication. Outcome assessors and statistical analysts will be blind to the procedure and results of randomization, group allocation, and intervention.

\section{Interventions}

Subjects receive regular medical therapy (Symbicort or Seretide) as usual while participating in this study, except that it is not allowed to change the asthma control medication during this study. Based on the regular therapy, asthmatic patients randomly allocated will receive two different acupuncture treatments: puncturing at acupoints on the Lung Meridian or acupoints on the Heart Meridian.

\section{Acupuncture intervention}

According to records in the ancient Traditional Chinese Medicine work Huangdi Neijing, acupuncture points belonging to Lung Meridian have a noticeable effect on Lung disease. Therefore, we chose the following acupuncture points: patients in group A will receive manual acupuncture at six acupoints belong to Lung Meredian (bilateral Taiyuan (LU9), Lieque (LU7), Chize (LU5)) with disposable sterile filiform needles $(0.25 \times 25 \mathrm{~mm}, 0.35 \times 40 \mathrm{~mm}$, Huatuo Medical Instrument Co., Ltd., China). Patients in group $B$ is the reference group, will receive manual acupuncture at six acupoints belong to Heart Meredian (bilateral Shenmen (HT7), Yinxi (HT6), Shaohai (HT3). The manipulations are as follows: needles will be inserted into acupoints at a depth of 20-30 mm after skin disinfection using alcohol; acupuncturists will then bi-directionally twist needles by $90^{\circ}-180^{\circ}$, lifting and thrusting needles with the amplitude of $3-5 \mathrm{~mm}$ for $1-1.5 \mathrm{~Hz}$ to induce deqi sensation. After the deqi sensation is attained, needles will be retained at the acupoints for $30 \mathrm{~min}$. During the $30 \mathrm{~min}$, the above procedures will be manipulated intermittently to maintain the deqi sensation.

Patients both in the two groups will receive a total of 12 sessions of acupuncture in four weeks with three sessions per week. All the acupuncture manipulation will be performed by two licensed acupuncturists with at least three years of clinical experience. 
The acupuncture treatment will be conducted by doctors of department of Acupuncture and Tuina School of CDUTCM, with more than 6 years of Traditional Chinese medicine college education and at least 4 years of clinical experience.

\section{Regular medical therapy}

During the study period, all participants will receive regular medical therapies according to the Chinese guideline for the prevention and management of bronchial asthma. Asthma medications, such as sodium cromolyn, nedocromil sodium, leukotriene modifiers, and theophylline, require a stable dose for 3 months before enrollment; Nasal glucocorticoids and antihistamines require a stable dose for 2 months before enrollment; A short course of antihistamines or nasal corticosteroids is allowed to treat pollinosis; Desensitization treatment in the maintenance period requires a stable dose for at least 1 month before enrollment. During the study, short-term (<10 days) use of systemic corticosteroids and temporary use of aerosolized therapy (including beta 2-agonists, anticholinergics, and steroids) and antibiotic therapy were allowed for acute asthma attacks. All medications used need to be maintained at a stable dose throughout the study period. Furthermore, a salbutamol metered-dose inhaler (100 mg per puff) will be provided as rescue medication throughout the trial.

\section{MRI data acquisition}

MRI data will be acquired with a 3.0-T MR scanner (Siemens AG, Germany) at Huaxi Magnetic Resonance Research Center, West China Hospital of Sichuan University, Chengdu, China. All patients will be asked to stay awake, keep their eyes open, and remain still at the stage of the fMRI can. The participant' head should be placed in the head mask and a sponge will be inserted to strengthen the fixation of the head. The scanning procedure contains a localizer, a high-resolution three-dimensional T1-weighted imaging (3D-T1WI), a blood oxygenation level-dependent fMRI (BOLD-fMRI) and a diffusion tensor imaging (DTI) sequence. The 3D-T1WI scanning parameters will be as follows: repetition time (TR)/echo time $($ TE) = 1900/2.26ms; slice thickness = $1 \mathrm{~mm}$; slice number = 30; matrix size= $128 \times 128$; and field of view (FOV) $=256 \times 256 \mathrm{~mm}$. The BOLD-fMRI scanning parameters will be as follows: TR/TE = 2000/30 ms; flip angle $=90^{\circ}$; slice number $=30 ;$ matrix size $=128 \times 128 ; \mathrm{FOV}=240 \times 240 \mathrm{~mm}$; slice thickness $=5 \mathrm{~mm}$; and total volume $=240$. The DTI data will be acquired with the following parameters: FOV $=240 \times 240$ $\mathrm{mm} ; \mathrm{TR} / \mathrm{TE}=6800 / 93 \mathrm{~ms}$; matrix size $=128 \times 128$; and slice thickness $=3 \mathrm{~mm}$ with no gap. Two diffusion-weighted sequences were acquired using gradient values $b=1000 \mathrm{~s} / \mathrm{mm} 2$ and $b=0$ with the diffusion-sensitizing gradients applied in 64 non-collinear directions. All images will be checked by a consultant radiologist at West China Hospital of Sichuan University to exclude unexpected brain lesions in recruits.

\section{Outcomes}

The primary outcome measurements are the Asthma Quality of Life Questionnaire (AQLQ) [21], for assessing health-related impairment of quality of life in adult asthmatic patients [22]. AQLQ contains 32 
items which including four domains: activity limitation, symptoms, mental health, environmental stimuli. It will take 5-10 minutes to the assessor.

The secondary outcome measurements including the Asthma Control Test (ACT), the Rate of Peak Expiratory Flow (PEFR), Forced Expiratory Volume in 1 second (FEV1), Montreal Cognitive Assessment (MoCA), Self-rating Anxiety Scale (SAS) and Self-rating Depression Scale (SDS). The ACT is a questionnaire consisting of five questions with a 5-point scale for each, which is validated for assessing asthma control [23]. PEFR and FEV1 are the main and objective monitors for asthma therapy.

Furthermore, this trial also selects SAS, SDS, and MoCA as the secondary clinical outcomes to assess the emotional state and the general cognitive function, for psychological cognition importance in asthma patient's pathogenesis.

All outcomes will be assessed at baseline and the end of treatment. The overview of the outcome measurement at different time points is shown in Table 1.

\section{Patient safety}

Safety monitoring will be conducted throughout the trial with reporting of adverse events (AEs) and serious adverse events (SAEs) in each participant's case report form (CRF). All AEs/SAEs will be reported immediately to the study principal investigator and attending physician. Each AE will be recorded separately.

\section{Data management}

Clinical data will be managed with printed and electronic CRFs. Only outcome assessors have access to CRFs and will perform double-data entry. The researchers will be required to follow the requirements of the case report form and fill in the relevant information in a timely and accurate manner. The Evidencebased Medicine Center of the CDUTCM will be responsible for monitoring the study and data every three months and will make the final decision to terminate the trial.

\section{Data analysis}

The primary analysis will be conducted on a per-protocol basis. The data analysis will be completed by statisticians who are independent of the research and blind to the group mark. For non-imaging data, statistical analyses will be conducted with SPSS 23.0 software (IBM Corporation, Armonk, NY, USA). For clinical information, data will be presented as means with SDs. Student T-test and Chi-Square test will be used to compare group differences at baseline. Paired t-test will be used to compare the clinical outcomes within the group. Analysis of variance and Kruskal-Wallis Test will be used to compare the clinical feature change between groups. The significance will be set at $5 \%$ level with two-side test. The clinical and neuropsychological data including AQLQ, ACT, MoCA, SAS, and SDS will be converted to domain z scores for correlational analysis with imaging data. 
The MRI data will be preprocessed and analyzed by SPM12 (http://www.fil.ion.ucl.ac.uk/spm/) and CONN toolbox 18b (https://web.conn-toolbox.org/) performed on MATLAB 2014b (MathWorks, Inc., Natick, MA, USA). The structural MRI data will analyze using VBM toolbox within SPM12. The steps including checking for artifacts, structural abnormalities, and pathologies; image segment; normalizing to standard template; spatial smoothing. The preprocessing steps of fMRI data including slice timing correction; head motion correction; skull-stripping using BET; co-registration of the anatomical image to the mean functional image; segmentation of the anatomical gray matter, white matter, and CSF; normalization to the MNI152 standard template; smoothing; Band-pass filtering. After preprocessing, a series of brain activity information including amplitude of low-frequency fluctuation (ALFF), group Independent Component Analyses (ICA), seed-based functional connectivity, complex-network analyses, and dynamic connectivity analyses, will be calculated to investigate the brain response for different treatment. Finally, the Pearson correlation analysis will be used to assess the association between the changes of cerebral activity features and the improvement of clinical outcomes in each group.

\section{Discussion}

Established on the "meridians-viscera relationship" theory of traditional Chinese medicine and modern central mechanism of acupuncture the present randomized controlled neuroimaging study is the first one to explore whether and how specific meridian acupuncture works in improving quality of life and symptoms by modulating the altered brain function in asthma patients.

\section{Study feasibility}

In recent years, brain imaging techniques had been applied to investigate the physiopathology of asthma and revealed that the structural and functional abnormalities in brain circuits involved in the pathogenesis of asthma [13-17, 24]. These studies provided an approach to investigate the potential mechanism of some intervention for asthma control. Subsequently, a few longitudinal studies have begun to uncover functional alterations in the brain due to treatment for asthma. For instance, Zhang et al. [25] investigated the spontaneous brain activity with resting-state fMRI before and after cognitivebehavioral therapy (GCBT) in adult asthmatic patients. They reported that the beneficial effects of GCBT on asthma control level were associated with the reversed abnormal spontaneous brain activity in the bilateral occipital lobe and sensorimotor cortex.

The central integration has been considered as an essential mechanism for acquiring acupuncture therapeutic effect since the 1970s [26, 27]. In the past 20 years, with the aid of functional brain imaging techniques, such as fMRI, positron emission tomography (PET), electroencephalography (EEG), and magnetoencephalography (MEG)[28], investigators mapped the cerebral regions/circuit/networks participating in acupuncture effects for treating neuropathies [29-32], gastrointestinal disorders [33-35], motor diseases $[36,37]$. These studies provided abundant visualized evidence for understanding the central mechanism of acupuncture. 
Although acupuncture-related neuroimaging studies of asthmatic patients have not yet been covered, the proven central pathological features of asthma and the rich experience of acupuncture-neuroimaging studies all provide a basis for investigating the central mechanism of acupuncture treating asthma in this study.

This study is concerned with the relative specificity of the "meridians-viscera correlation", and expected to provide evidence for the optimization of acupuncture treatment for asthma. In traditional meridian theories, "meridians-viscera correlation " is an essential principle for acupuncture treating viscera-related diseases [38]. It means that each meridian pertains to some specific viscera and connects with several visceras. When the viscera are affected, the acupoints on the related-meridian can be selected for the affected viscera. for example, the Lung meridian pertains to Lung, so the acupoints on the Lung Meridian can be used for lung diseases. The "meridians-viscera correlation " theory has also been proven by modern clinic trails. Our previous RCT demonstrated that, compared with acupoint on the gallbladder meridian, acupuncturing at stomach Meridian showed a better effect in improving QoL and relieving symptoms for patients with functional dyspepsia (FD) [39], indicating that the Stomach Meridian is the best choice for treating stomach disease. Our later neuroimaging studies [40] also demonstrated that puncturing at the acupoints on Stomach meridian elicited wider and more remarkable positive modulation on the abnormal cerebral function in FD patients than that on Gallbladder meridian. So, this study focusses on the difference in efficacy and cerebral responses resulted from different acupuncture intervention, and try to provide evidence for the "meridians-viscera correlation" theory.

\section{The quality control program}

The quality control program is the precondition for the result reliability. Our previous study indicated that strict quality control plays an essential role in the guarantee of high repeatability in acupunctureneuroimaging studies [41]. Accordingly, we designed a quality control program focused on the homogeneity of participants, standardization of fMRI scan, and strictness of acupuncture procedure, to improve the reproducibility and reliability of the result.

First, one of the important considerations in the neuroimaging study is that the neural activity of human beings is highly variable. The connectivity topology of the brain can be influenced by potentially confounding factors, such as age [42], gender [43], handedness [44], and even mental state [45]. Hence, rigorous inclusion/exclusion criteria at the stage of participant enrollment will be established in this study. For example, asthma patients will be restricted to age between 40 to 65 years and being righthanded to guarantee the homogeneous clinical populations. Concurrently, the SAS and SDS scores will be recorded to evaluate the patients' psychological states and rule outpatients with severe anxiety and depression.

The sensitivity to subject motion is another challenge in functional MRI studies [46]. Even the slightest source of artifacts such as physiological fluctuations (respiration and cardiac fluctuations) as well as head motion can highly influence the final estimates of connectivity. Hence, the patients will be asked to stay awake, keep their eyes open, and remain still at the stage of the fMRI scan. To eliminate possible 
head motion, participant' head should be placed in the head mask and a sponge will be inserted to strengthen the fixation of the head.

Finally, the acupuncture manipulation will be performed by two experienced acupuncturists with a strict and standard operation procedure. The Deqi sensation will be evaluated and recorded with the VAS after each acupuncture treatment.

The limitations of this study lie primarily in the seasonality of asthma attacks [47] and the diversity of routine medical treatment for patients with asthma [48]. Thus, the inconsistent regular treatments of asthma may be a confounding of our results.

In summary, this fMRI trial is designed to investigate the clinical and cerebral activity changes evoked by different meridians (the acupoints on the Lung Meridian vs. the acupoints on the Heart Meridian) on patients with mild to moderate, persistent asthma, and explore the associations between the cerebral activity changes and clinical variable changes. The results of this trial will help to provide the optimal adjunct approach for improving the quality of life in adults with persist asthma and provide visualization evidence for the clinical application of acupuncture for asthma management.

\section{Trial status}

This trial registered on Chinese Clinical Trial Registry (https:// www.chictr.org.cn) on 15 November 2019 (Registered number: ChiCTR1900027478, the protocol version number: V2.0). The patient recruitment of this trial has not been started and will begin on December 1, 2019.

\section{Abbreviations}

ACC: Anterior Cingulate Cortex; ACT: Asthma Control Test; AEs: Adverse Events; ALFF: Amplitude of Lowfrequency Fluctuation; AQLQ: Asthma Quality of Life Questionnaire; BOLD-fMRI: Blood Oxygenation Level-dependent fMRI; CDUTCM: Chengdu University of Traditional Chinese Medicine; CRF: Case Report Form; DTI: Diffusion Tensor Imaging; 3D-T1WI:Three-dimensional T1-weighted Imaging; EEG:

Electroencephalography; FD: Functional Dyspepsia; FEV1: Forced Expiratory Volume in 1 second; GCBT: Cognitive Behavioral Therapy; GINA: Global Initiative for Asthma; ICA: Independent Component Analyses; ICS: Inhaled Corticosteroids; Ig E: Immunoglobulin E; IRB: Institutional Review Board; LABA: Long-acting B2 Agonist; MEG: Magnetoencephalography; MoCA: Montreal Cognitive Assessment; MRI: Magnetic Resonance Imaging; PEFR: the Rate of Peak Expiratory Flow; PET: Positron Emission Tomography; RCTs: Randomized Controlled Trials; SAEs: Serious Adverse Events; SAS: Self-rating Anxiety Scale; SDS: Selfrating Depression Scale; VAS: Visual Analogue Score.

\section{Declarations}

\section{Acknowledgments}


We thank Qingsong Huang, Yan Dong, Rong Pu, Wei Xiao, Wujun Wang and Jianyun Jiang, Departments of Respiratory, the First Affiliated Hospital of CDUTCM, for the help of participants enrollment.

\section{Funding}

The trial is financially supported by the National Key Research and Development Program (NO.2018YFC1704600, 2018YFC1704605).

\section{Author information}

Siyi Yu, Xiaohui Dong, and Ruirui Sun ${ }^{1}$ contributed equally to this work.

\section{Affiliations}

Brain Research Center, Acupuncture and Tuina School/The 3rd Teaching Hospital, Chengdu University of Traditional Chinese Medicine, Chengdu 610075, Sichuan, China.

Siyi Yu, Xiaohui Dong, Ruirui Sun, Zhaoxuan He, Xiaojuan Hong, Lei Lan \& Fang Zeng

Hospital of Chengdu University of Traditional Chinese Medicine, Chengdu 610072, Sichuan, China

Chuantao Zhang

The Fifth People's Hospital of Chengdu, Chengdu 611130, Sichuan, China

Mei Chen

\section{Contributions}

FZ and LL are responsible for this study. FZ, LL, RS, ZH, and XH conceived and designed the study. SY, $X D$, and $R S$ participated in drafting the trial protocol and preparing the manuscript. $S Y, X D, Z H, C Z, M C$, and $\mathrm{XH}$ participated in data collection and were in charge of recruitment and treatment of patients. All authors read and approved the final manuscript.

\section{Corresponding authors}

Correspondence to Lei Lan or Fang Zeng.

\section{Ethics declarations}

\section{Ethics approval and consent to participate}

This study will be performed according to the guidance and principles of the Declaration of Helsinki. Ethics approval for this trial has been granted by the Institutional Review Board (IRB) the First Affiliated Hospital of CDUTCM (approved number. 2019KL-045). All participants will provide voluntary written informed consent after a full discussion about the potential benefits and risks before participation. 


\section{Consent for publication}

Not applicable.

\section{Competing interests}

The authors declare that they have no competing interests.

\section{References}

1. Masoli M, Fabian D, Holt S, Beasley R, Program GIfA. The global burden of asthma: executive summary of the GINA Dissemination Committee report. Allergy. 2004;59:469-78.

2. Heaney LG, Robinson DS. Severe asthma treatment: need for characterising patients. The Lancet. 2005;365:974-6.

3. Papi A, Brightling C, Pedersen SE, Reddel HK. Asthma. Lancet (London, England). 2018;391:783-800.

4. Calhoun WJ, Haselkorn T, Mink DR, Miller DP, Dorenbaum A, Zeiger RS. Clinical burden and predictors of asthma exacerbations in patients on guideline-based steps 4-6 asthma therapy in the TENOR cohort. The Journal of Allergy and Clinical Immunology: In Practice. 2014;2:193-200. e3.

5. Brinkhaus B, Roll S, Jena S, Icke K, Adam D, Binting S, et al. Acupuncture in patients with allergic asthma: A randomized pragmatic trial. The Journal of Alternative and Complementary Medicine. 2017;23:268-77.

6. Choi JY, Jung HJ, Kim JI, Lee MS, Kang KW, Roh YL, et al. A randomized pilot study of acupuncture as an adjunct therapy in adult asthmatic patients. The Journal of asthma : official journal of the Association for the Care of Asthma. 2010;47:774-80.

7. Biernacki W, Peake MD. Acupuncture in treatment of stable asthma. Respiratory medicine. 1998;92:1143-5.

8. Reinhold T, Brinkhaus B, Willich SN, Witt C. Acupuncture in patients suffering from allergic asthma: is it worth additional costs? Journal of alternative and complementary medicine (New York, NY). 2014;20:169-77.

9. Chu KA, Wu YC, Ting YM, Wang HC, Lu JY. Acupuncture therapy results in immediate bronchodilating effect in asthma patients. Journal of the Chinese Medical Association : JCMA. 2007;70:265-8.

10. Pai HJ, Azevedo RS, Braga AL, Martins LC, Saraiva-Romanholo BM, Martins Mde A, et al. A randomized, controlled, crossover study in patients with mild and moderate asthma undergoing treatment with traditional Chinese acupuncture. Clinics (Sao Paulo, Brazil). 2015;70:663-9.

11. Jiang C, Jiang L, Qin Q. Conventional Treatments plus Acupuncture for Asthma in Adults and Adolescent: A Systematic Review and Meta-Analysis. Evidence-based complementary and alternative medicine : eCAM. 2019;2019:9580670.

12. Busse WW. The brain and asthma: what are the linkages? Allergy and the Nervous System. 98: Karger Publishers; 2012. p. 14-31. 
13. von Leupoldt A, Sommer T, Kegat S, Eippert F, Baumann HJr, Klose H, et al. Down-regulation of insular cortex responses to dyspnea and pain in asthma. American journal of respiratory and critical care medicine. 2009;180:232-8.

14. Rosenkranz MA, Busse WW, Johnstone T, Swenson CA, Crisafi GM, Jackson MM, et al. Neural circuitry underlying the interaction between emotion and asthma symptom exacerbation. Proceedings of the National Academy of Sciences. 2005;102:13319-24.

15. Bian R, Zhang Y, Yang Y, Yin Y, Zhao X, Chen H, et al. White Matter Integrity Disruptions Correlate With Cognitive Impairments in Asthma. Journal of magnetic resonance imaging : JMRI. 2018.

16. Li Q-G, Zhou F-Q, Huang X, Zhou X, Liu C, Zhang T, et al. Alterations of resting-state functional network centrality in patients with asthma: evidence from a voxel-wise degree centrality analysis. NeuroReport. 2018;29:1151-6.

17. Gao X, Xiao Y, Lv P, Zhang W, Gong Y, Wang T, et al. Altered brain network integrity in patients with asthma: A structural connectomic diffusion tensor imaging study. Respiratory physiology \& neurobiology. 2019;266:89-94.

18. Chan AW, Tetzlaff JM, Altman DG, Laupacis A, Gøtzsche PC, Krleža-Jerić K, et al. SPIRIT 2013 statement: defining standard protocol items for clinical trials. Ann Intern Med. 2013;158:200-7.

19. Workgroup Asthma CTS. Chinese guideline for the prevention and management of bronchial asthma (Version 2016). Chinese journal of tuberculosis and respiration. 2016;39:675-97.

20. Desmond JE, Glover GH. Estimating sample size in functional MRI (fMRI) neuroimaging studies: statistical power analyses. Journal of neuroscience methods. 2002;118:115-28.

21. Juniper EF, Guyatt G, Epstein R, Ferrie PJ, Jaeschke R, Hiller T. Evaluation of impairment of health related quality of life in asthma: development of a questionnaire for use in clinical trials. Thorax. 1992;47:76-83.

22. Testa MA, Simonson DC. Assessment of quality-of-life outcomes. New Engl J Med. 1996;334:835-40.

23. Nathan RA, Sorkness CA, Kosinski M, Schatz M, Li JT, Marcus P, et al. Development of the asthma control test: a survey for assessing asthma control. Journal of Allergy and Clinical Immunology. 2004;113:59-65.

24. Xiong $X$, Zhu H, Wang T, Ji Y. Altered intrinsic regional brain activity in female asthmatics with or without depressive symptoms: A resting-state functional magnetic resonance imaging study. The Journal of asthma : official journal of the Association for the Care of Asthma. 2016;53:922-9.

25. Yu SY, Lv ZT, Zhang Q, Yang S, Wu X, Hu YP, et al. Electroacupuncture is Beneficial for Primary Dysmenorrhea: The Evidence from Meta-Analysis of Randomized Controlled Trials. Evidence-based complementary and alternative medicine : eCAM. 2017;2017:1791258.

26. Han JS, Ho YS. Global trends and performances of acupuncture research. Neuroscience and biobehavioral reviews. 2011;35:680-7.

27. Yang ES, Li PW, Nilius B, Li G. Ancient Chinese medicine and mechanistic evidence of acupuncture physiology. Pflugers Archiv : European journal of physiology. 2011;462:645-53. 
28. He T, Zhu W, Du SQ, Yang JW, Li F, Yang BF, et al. Neural mechanisms of acupuncture as revealed by fMRI studies. Autonomic neuroscience : basic \& clinical. 2015;190:1-9.

29. Napadow V, Makris N, Liu J, Kettner NW, Kwong KK, Hui KK. Effects of electroacupuncture versus manual acupuncture on the human brain as measured by fMRI. Human brain mapping. 2005;24:193205.

30. Duan G, He Q, Pang Y, Chen W, Liao H, Liu H, et al. Altered amygdala resting-state functional connectivity following acupuncture stimulation at BaiHui (GV20) in first-episode drug-Naïve major depressive disorder. Brain Imaging Behav. 2019.

31. Li Y, Wang Y, Liao C, Huang W, Wu P. Longitudinal Brain Functional Connectivity Changes of the Cortical Motor-Related Network in Subcortical Stroke Patients with Acupuncture Treatment. Neural plasticity. 2017;2017:5816263.

32. Wu P, Zhou YM, Liao CX, Tang YZ, Li YX, Qiu LH, et al. Structural Changes Induced by Acupuncture in the Recovering Brain after Ischemic Stroke. Evidence-based complementary and alternative medicine : eCAM. 2018;2018:5179689.

33. Zeng F, Qin W, Ma T, Sun J, Tang Y, Yuan K, et al. Influence of acupuncture treatment on cerebral activity in functional dyspepsia patients and its relationship with efficacy. The American journal of gastroenterology. 2012;107:1236-47.

34. Zhou S, Zeng F, Liu J, Zheng H, Huang W, Liu T, et al. Influence of acupuncture stimulation on cerebral network in functional diarrhea. Evidence-based complementary and alternative medicine : eCAM. 2013;2013:975769.

35. Liu CZ, Xie JP, Wang LP, Liu YQ, Song JS, Chen YY, et al. A randomized controlled trial of single point acupuncture in primary dysmenorrhea. Pain medicine (Malden, Mass). 2014;15:910-20.

36. Kong J, Wang Z, Leiser J, Minicucci D, Edwards R, Kirsch I, et al. Enhancing treatment of osteoarthritis knee pain by boosting expectancy: A functional neuroimaging study. Neurolmage Clinical. 2018;18:325-34.

37. Chen J, Wang Z, Tu Y, Liu X, Jorgenson K, Ye G, et al. Regional Homogeneity and Multivariate Pattern Analysis of Cervical Spondylosis Neck Pain and the Modulation Effect of Treatment. Frontiers in neuroscience. 2018;12:900.

38. Zhou Y, Wang K, Ling H, Zhou M, Wu Z, Cai R, et al. Meridian-Viscera Correlationship2013.

39. Ma TT, Yu SY, Li Y, Liang FR, Tian XP, Zheng H, et al. Randomised clinical trial: an assessment of acupuncture on specific meridian or specific acupoint vs. sham acupuncture for treating functional dyspepsia. Aliment Pharmacol Ther. 2012;35:552-61.

40. Zeng F, Lan L, Tang Y, Liu M, Liu X, Song W, et al. Cerebral responses to puncturing at different acupoints for treating meal-related functional dyspepsia. Neurogastroenterology and motility : the official journal of the European Gastrointestinal Motility Society. 2015;27:559-68.

41. Qiu K, Jing M, Sun R, Yang J, Liu X, He Z, et al. The status of the quality control in acupunctureneuroimaging studies. Evidence-Based Complementary and Alternative Medicine. 2016;2016. 
42. Nobis L, Manohar SG, Smith SM, Alfaro-Almagro F, Jenkinson M, Mackay CE, et al. Hippocampal volume across age: Nomograms derived from over 19,700 people in UK Biobank. Neurolmage: Clinical. 2019:101904.

43. Zeng F, Sun R, He Z, Chen Y, Lei D, Yin T, et al. Altered Functional Connectivity of the Amygdala and Sex Differences in Functional Dyspepsia. Clinical and translational gastroenterology. 2019;10:e00046.

44. Hatta T. Handedness and the brain: a review of brain-imaging techniques. Magnetic Resonance in Medical Sciences. 2007;6:99-112.

45. Lichenstein SD, Verstynen T, Forbes EE. Adolescent brain development and depression: A case for the importance of connectivity of the anterior cingulate cortex. Neuroscience and biobehavioral reviews. 2016;70:271-87.

46. Havsteen I, Ohlhues A, Madsen KH, Nybing JD, Christensen H, Christensen A. Are Movement Artifacts in Magnetic Resonance Imaging a Real Problem?-A Narrative Review. Frontiers in neurology. 2017;8:232.

47. Cohen HA, Blau H, Hoshen M, Batat E, Balicer RD. Seasonality of asthma: a retrospective population study. Pediatrics. 2014;133:e923-e32.

48. Low I, Wei SY, Lee PS, Li WC, Lee LC, Hsieh JC, et al. Neuroimaging Studies of Primary Dysmenorrhea. Advances in experimental medicine and biology.1099:179-99.

\section{Figures}




\section{Enrollment, evaluation, and consent of asthma patients}

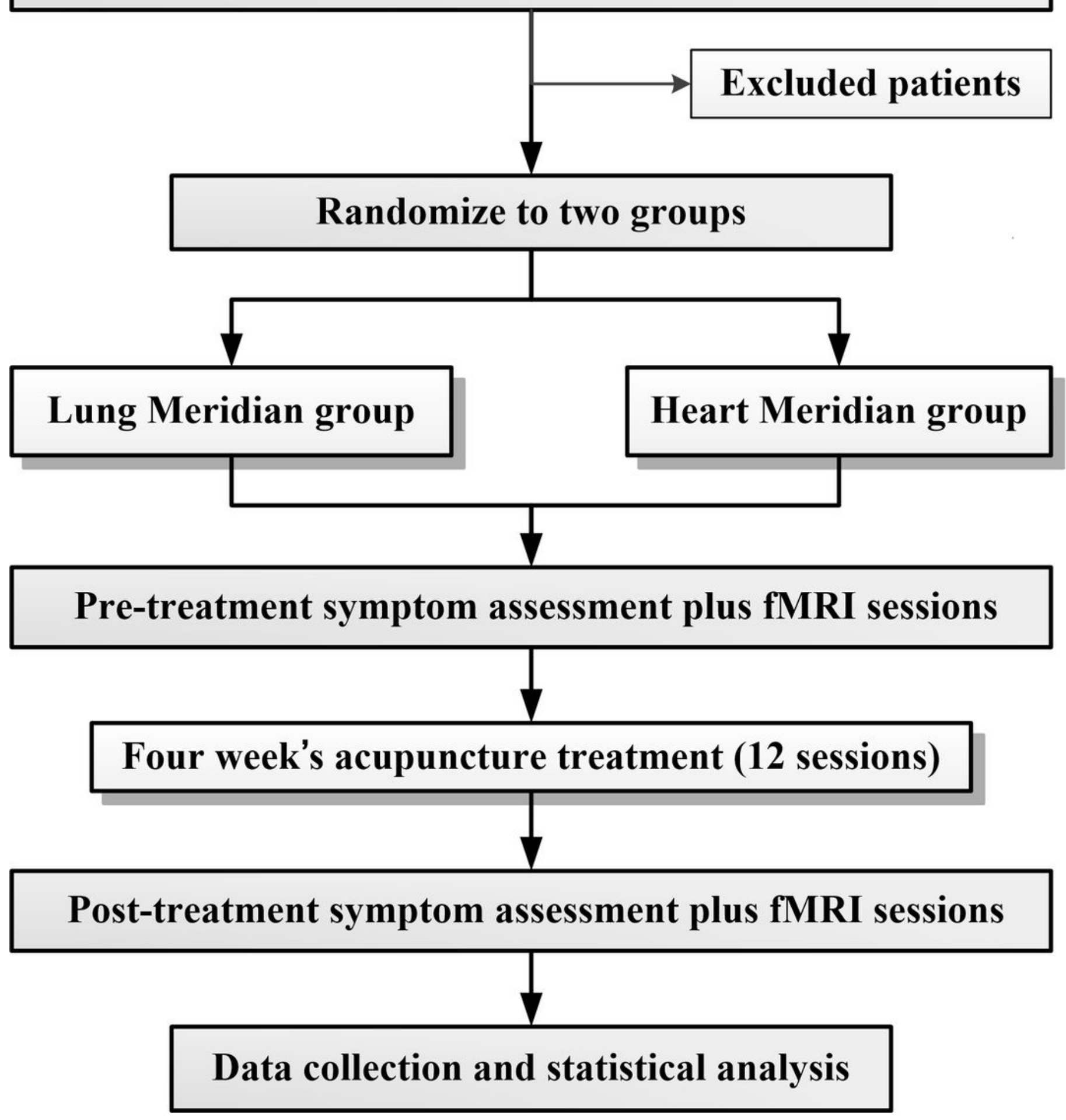

Figure 1

Flowchart of the trial. The present study is a randomized controlled neuroimaging trial. A total of 48 eligible patients will be randomized equally to two groups: Lung Meridian group, and Hear Meridian group. During a 4-week treatment period, patients in the two acupuncture groups will receive 12 sessions of puncturing treatments. Both the outcome assessments and functional magnetic resonance imaging (fMRI) scan will be performed at two-time points: baseline and end of acupuncture treatments. The 
central mechanism of acupuncture in the treatment of asthma will be analyzed after data collection. Abbreviations: fMRI functional magnetic resonance imaging.

\section{Supplementary Files}

This is a list of supplementary files associated with this preprint. Click to download.

- SPIRITFillablechecklist.doc

- SPIRITTCM2018.docx 